\title{
Effect of Adsorbed Helium on Electron Tunneling between Metal Electrodes
}

\author{
R. J. P. Keijsers, ${ }^{1}$ J. Voets, ${ }^{1}$ O. I. Shklyarevskii, ${ }^{1,2}$ and H. van Kempen ${ }^{1}$ \\ ${ }^{1}$ Research Institute for Materials, University of Nijmegen, Toernooiveld 1, 6525 ED Nijmegen, The Netherlands \\ ${ }^{2}$ B. Verkin Institute for Low Temperature Physics and Engineering, National Academy of Science of Ukraine, 47 Lenin Avenue, \\ 310164 Kharkov, Ukraine
}

(Received 31 July 1995)

\begin{abstract}
The tunnel resistance of highly stable mechanically controlled break junctions of $\mathrm{Al}, \mathrm{Pt}, \mathrm{Ag}$, and $\mathrm{Au}$, recorded as a function of the electrode spacing, is found to be strongly influenced by the presence of adsorbed He atoms at low temperatures. Telegraph-noise-like resistance fluctuations of the tunnel resistance occur in a certain range of electrode separations when the measurements are performed in He gas at $T=1.2 \mathrm{~K}$. Some models which may be capable of explaining the observed effects are discussed.
\end{abstract}

PACS numbers: $73.40 . \mathrm{Gk}$

Rare gas atoms adsorbed on metal surfaces were long expected to be invisible in electron tunneling experiments, since they would make hardly any contribution to the state density at the Fermi level. But a few years ago, Eigler et $a l$. presented clear scanning tunneling microscope (STM) images of $\mathrm{Xe}$ adsorbed on a $\mathrm{Ni}(110)$ surface [1], and it was demonstrated that it was possible to move these atoms individually to chosen positions on the surface [2]. It was calculated that the broadened $6 s$ resonance of $\mathrm{Xe}$, even though it is virtually unfilled, leads to a redistributed conduction-electron density at the Fermi level which extends further into the vacuum than in the case of a bare metal surface $[1,3]$.

The smallest rare gas atom, helium, has also been extensively studied, mainly because of its special lowtemperature properties. Calculations by Lang [4], similar to the calculations for Xe mentioned above, have shown that the closed valence shell of an adsorbed $\mathrm{He}$ atom polarizes metal states away from the Fermi level, leading to a local decrease in the state density. Therefore, an adsorbed $\mathrm{He}$ atom should occur in an STM image as a small dip in the surface. Yet, as far as we know, helium has never been reported to influence the electron tunneling between two metallic electrodes. A number of low-temperature STMs that have been constructed lately often even use helium as a contact gas [5], implicitly assuming that the helium will not disturb the measured images.

We report here a study of the tunnel current (or resistance) as a function of electrode separation using highly stable mechanically controllable break (MCB) junctions. The basic idea of the MCB junction technique is to break a thin metallic wire at low temperatures in a high vacuum environment, thus creating two clean electrodes which can be used for point-contact or tunneling experiments. To this extent, the wire is fixed onto a phosphor-bronze bending beam, covered with a thin insulating foil, at two closely spaced points. The wire is deeply notched in between these anchoring points, and is broken by bending the beam (see inset of Fig. 1). The electrodes are then displaced with respect to each other by changing the bending angle. This sample mounting ensures a very high stability, and an accurate adjustability of the electrode separation. For a more detailed description of the MCB technique and the specific setup used here we refer to [6-8]. When the experiments were performed in vacuum at low temperatures (we estimate a residual pressure of $10^{-12}-10^{-14}$ Torr at $4.2 \mathrm{~K}$ ), almost always perfect exponential behavior was observed (curve Pt-1 of Fig. 1). The presence of even very small amounts of ${ }^{4} \mathrm{He}$ or ${ }^{3} \mathrm{He}$ gas was found to cause a clear and reproducible deviation from this exponential behavior around tunnel resistance values of 100 to $1000 \mathrm{M} \Omega$, which is in the common range of STM operation. This deviation may therefore lead to

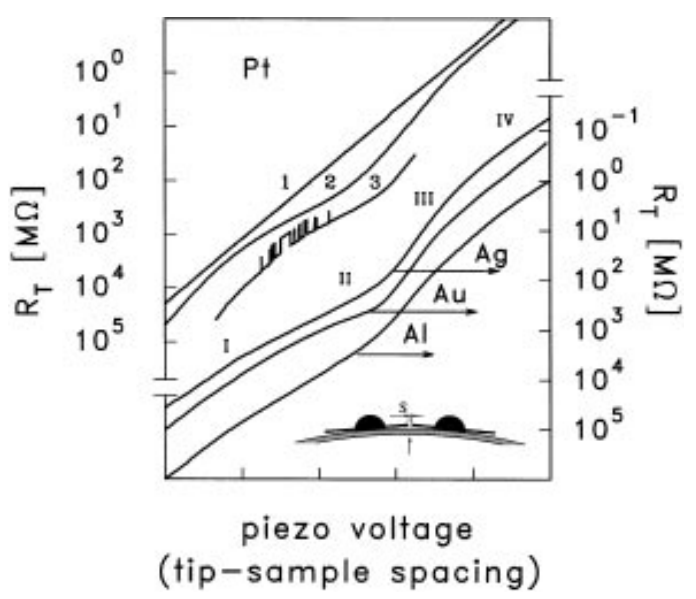

FIG. 1. Tunnel resistance as a function of piezovoltage (proportional to electrode separation) for MCB junctions in vacuum (Pt-1), or in a low-pressure ${ }^{4} \mathrm{He}$ gas environment at $T=4.2$ (Au, Ag, Al, and Pt-2) and $1.2 \mathrm{~K}(\mathrm{Pt}-3)$. The bias voltage across the junction is $100 \mathrm{mV}$. At resistance values of 100 to $1000 \mathrm{M} \Omega$, clear deviations from the usual exponential behavior in vacuum appear when the experiment is performed in a ${ }^{4} \mathrm{He}$ gas environment. Additionally, discrete resistance changes start to occur as the temperature is lowered to $1.2 \mathrm{~K}$. Four distance regimes, discussed in the text, are indicated by I-IV. The inset shows a schematic drawing of the MCB sample design. 
severe mistakes, e.g., because of an error in calibrating an STM, but also when one is measuring the work function of a material.

Figure 1 displays the tunnel resistance $R_{T}$ of $\mathrm{Au}, \mathrm{Ag}$, $\mathrm{Al}$, and Pt (curve Pt-2) MCB junctions in a low-pressure, high-purity $(99.999 \%){ }^{4} \mathrm{He}$ gas environment at $4.2 \mathrm{~K}$ as a function of the voltage $V_{p}$ applied to the piezo driver. The electrode separation is proportional to this piezo voltage, but the proportionality factor may differ from sample to sample, which makes it difficult to calibrate the obtained displacements [8]. The horizontal axis for the measurements displayed in Fig. 1 has for this reason been rescaled in such a way that all curves cover approximately the same electrode separation range, which we estimate to start at $8-10 \AA$ at the left side of the plot, decreasing to about $2 \AA$, close to the jump to contact, at the right side. The observed deviation from exponential behavior can be described as a reduction of the decrease of the tunnel resistance at certain electrode separations, more or less recovering to the initial exponential behavior at even smaller distances. The same effect occurred when using ${ }^{3} \mathrm{He}$ as a contact gas. The shape of the distorted $R_{T}\left(V_{p}\right)$ curves did not change much for different helium pressures within the range of 0.01-760 Torr at $4.2 \mathrm{~K}$, and was even approximately the same for curves recorded with the junction directly immersed in liquid helium. It is therefore very likely that the effect is related to the first monolayer(s) of adsorbed He atoms, whereas the presence of the free exchange gas has no significant influence.

For a qualitative explanation of the observed behavior of $R_{T}\left(V_{p}\right)$, we will consider three ranges for the distance at the point of smallest separation of the two electrodes. At very small distances, there is no room for a $\mathrm{He}$ atom between the electrodes. In the next distance range, only one adsorbed He layer can be present. Because it is energetically more favorable to be adsorbed on a flat electrode than on protruding atoms, we will assume that in this range the adsorbed layer is situated at the electrode which is the flattest of the two in the region around the point of smallest separation. In accordance with STM terminology, we will refer to this electrode as the "sample," while the other, sharper electrode will be called "tip." (The assumption that at least one of the broken electrodes is rather sharp on an atomic scale in the region around the point of smallest separation is supported by the fact that one-atom contacts can be established in a simple manner using MCB junctions [9].) The third distance range is the one where the distance between the electrodes is so large that there is room for two or more monolayers of adsorbed He atoms. Calculations have shown that the equilibrium distance of a He atom to smooth noble metal or aluminum surfaces is rather large (2.5-3.0 [10] or $3.5 \AA$ [11]) for physically adsorbed He. Thus, no adsorbed He will be present for electrode separations below 3-4 $\mathrm{A}$, while a distance of
5-6 $\AA$ is required for one monolayer. For two or more layers, the separation should be larger than 10-12 $\AA$. Therefore, the experiment covers the ranges where there is no, or only one, layer of adsorbed He present between the electrodes. The calculated depth of the adsorption well varies between 5 and $10 \mathrm{meV}$ [10], but another calculation resulted in a much lower value $(1-2 \mathrm{meV})$ for the adsorption energy [11].

For the largest distances between tip and sample, the interaction between the He adsorbed at the sample and the front atom(s) of the tip is negligible, and $R_{T}$ decreases almost exponentially with decreasing electrode separation (part I of the curves in Fig. 1). When the electrodes are brought closer together, the polarizing effect on the tip of the He adsorbed at the sample side will become much stronger, leading to an increasing reduction of the tip density of states. This reduction results in a reduced tunnel probability and hence a slower decrease of $R_{T}$ with decreasing distance (part II in Fig. 1) in comparison to the unperturbed case. In region III, the total adsorption potential well, which has contributions from both electrodes, weakens, decreasing the probability for a He atom to be in the tunneling space. The local density of states (DOS) of tip and sample then recover within a small range of the electrode separation, leading to a rapid decrease of the tunnel resistance. The transition from region II to region III occurs in this model when the distance of the He to both tip and sample becomes close to the equilibrium distance to a single surface. Indeed, the estimated electrode separation of 5-6 $\AA$ at this point is only slightly larger than twice the theoretical equilibrium value. At even smaller distances (region IV), the situation is comparable to vacuum tunneling, and $R_{T}\left(V_{p}\right)$ shows exponential behavior again.

The calculations of Lang [4] for a He atom adsorbed at a Na surface show that the polarizing effect of the He on the local DOS will cause an STM to see a dip of about $0.3 \AA$ deep, when working in a constant current mode at a tip-sample distance of $8.5 \AA$. Since the latter value is only slightly larger than twice the equilibrium distance of an adsorbed $\mathrm{He}$ atom to a Na surface (4.1 $\AA$ according to [10]), the $0.3 \AA$ will probably be close to the maximum value obtainable. In the measurements of Fig. 1, the maximum deviation from exponential behavior amounts to about half a decade reduction in tunnel resistance. For the materials displayed here, one decade change in tunnel resistance corresponds to about $1 \AA$ change in electrode separation for clean surfaces. Thus, the experimental and theoretical values (however, obtained for different metals) are of comparable magnitude.

The shape of the curve does not change when the bias voltage is varied from 30 to $1000 \mathrm{mV}$, but at relatively low biases, the curve becomes more distorted, with the slope $d R_{T} / d s$ (where $s$ is the electrode spacing) getting close to zero for the lowest bias used here (Fig. 2). This bias voltage dependence may indicate that 


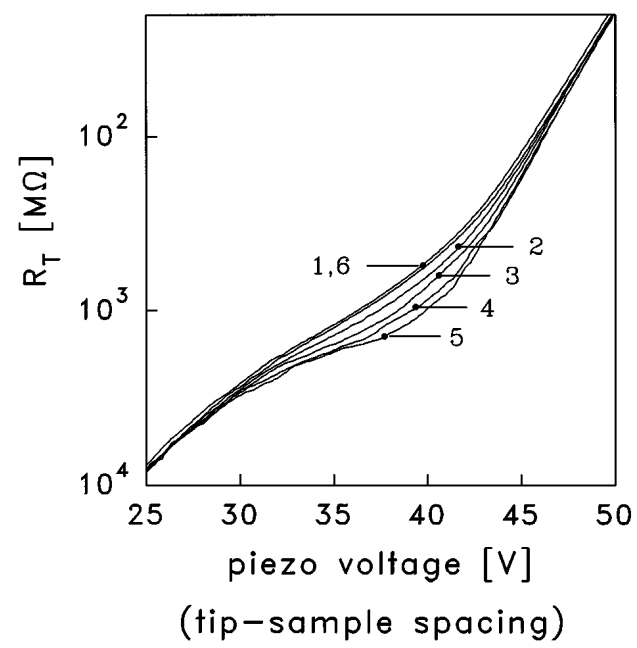

FIG. 2. Tunnel resistance as a function of piezo voltage for a Pt MCB junction in a ${ }^{4} \mathrm{He}$ gas environment at $T=4.2 \mathrm{~K}$, for different values of the bias voltage across the junction. Curves 1 and 6 were both recorded at a bias voltage of $15 \mathrm{mV}$, while the other four were recorded at bias voltages of (2) 10, (3) 6.7, (4) 4.5 , and (5) $3.0 \mathrm{mV}$. Curve 1 was recorded first, and curve 6 last, about $1 \mathrm{~h}$ later. The fact that these two curves almost coincide demonstrates the high sample stability.

an important contribution to the conduction electron state density reduction occurs in a rather narrow range of about $30 \mathrm{meV}$ around the Fermi level.

A very intriguing effect occurred when the measurements were performed at $T=1.2 \mathrm{~K}$. Part II of the curves in Fig. 1 became rather noisy and unstable, with sudden jumps of the tunnel resistance. A typical example of this behavior is shown in curve Pt-3 of Fig. 1. The number of jumps observed in such a curve increased when the curves were recorded at a lower speed. We therefore studied this instability by recording the tunnel resistance at fixed electrode separations as a function of time. It turned out that the tunnel resistance was jumping between two (but sometimes also three or four) distinct resistance values (Fig. 3). The relative change in resistance varied with distance, and could be as large as (30-40)\% for Au, and even up to $100 \%$ for Pt. Also, the switching rate varied with distance. This two-level resistance fluctuation resembles the atomic switch experiment of Eigler, Lutz, and Rudge [12], where a Xe atom could be reversibly moved between a tungsten STM tip and a nickel (110) surface by applying a voltage pulse in a limited junction resistance range. Here, the transfer of a xenon atom led to conductance changes up to a factor of 7 . It was shown that this transfer can be explained in terms of a single-atomtunneling process [13]. In our experiment, the resistance fluctuations occur spontaneously. The fluctuations can be explained in terms of a He atom tunneling between tip and sample, instantaneously causing a redistribution of the local DOS which is different for the two positions of the He. The fact that in our case the transition is sponta- neous may be due to a much larger tunneling probability of the He due to its lower mass, and a much lower barrier in the combined double-well adsorption potential of the two electrodes that occurs at certain tip-sample distances. However, for a better understanding more experimental information has to be obtained.

Of course, one can consider other mechanisms which may explain the observed behavior. For instance, one might be inclined to explain the switching between discrete resistance values by considering $\mathrm{He}$ atoms diffusing along the surface, changing the tunnel resistance whenever they enter or leave the tunnel space. The deviation from exponential behavior should then express a timeaveraged value of the discrete resistance values, in a situation where the time scale on which the changes occur is too fast to observe them separately. This model, however, does not apply, because it requires that one of the discrete resistance levels corresponds to the vacuum situation, and therefore one of the levels in the curve Pt-3 of Fig. 1 should follow an exponential behavior, which clearly is not the case. Also, it has been shown theoretically [14] that diffusion of atoms along a surface will mainly lead to an increase of the tunnel current noise, and will cause a very small increase of the tunnel resistance, hardly visible on a logarithmic scale.

In another model which may be capable to explain the observed effect, the He atom is regarded as a scattering center ("impurity") present in the tunneling space. As the electrode spacing is decreased, the $\mathrm{He}$ atom will block an increasing part of the solid angle in which the tunnel current flows, thus causing a slower decrease

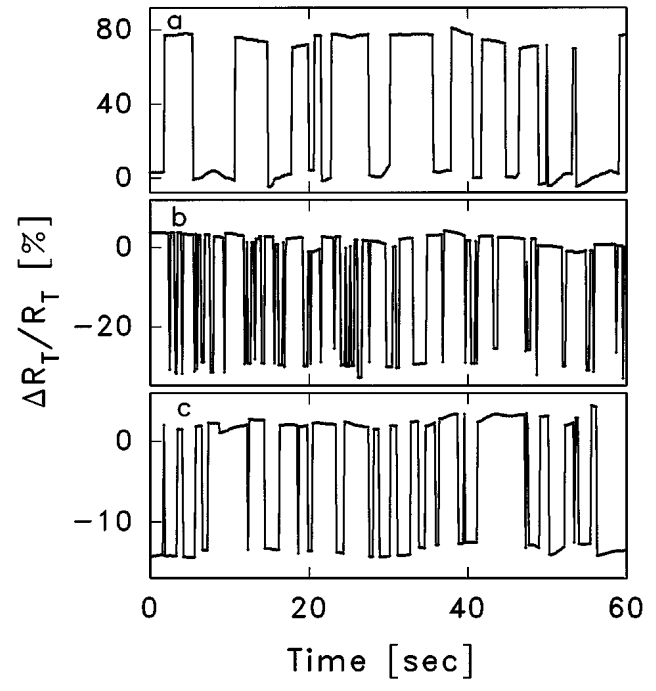

FIG. 3. Relative change in tunnel resistance as a function of time, for $\mathrm{MCB}$ junctions in a ${ }^{4} \mathrm{He}$ gas environment at $T=$ $1.2 \mathrm{~K}$, at different fixed electrode separations. The bias voltage across the junctions is $100 \mathrm{mV}$. The traces display jumps between two distinct resistance levels for a Pt junction, with (a) $R_{T}=4.8 \mathrm{G} \Omega$, (b) $R_{T}=1 \mathrm{G} \Omega$, and (c) $R_{T}=280 \mathrm{M} \Omega$. This type of noise is probably caused by He atoms tunneling between the two electrodes. 
of the tunnel resistance than in the case of vacuum tunneling. At short distances, the previously mentioned electron wind force will attempt to push the $\mathrm{He}$ atom out of the tunneling space. The switching behavior can be explained as a situation where there is a competition between the electron wind force, trying to shift the $\mathrm{He}$ out of the tunneling space, and the adsorption potential of the two-electrode system, which tries to pull the He in between the two electrodes. The fact that the shape of the distorted curves only changes for small biases (Fig. 2) can then be interpreted as the electron wind force passing a certain threshold value, above which the influence of the bias voltage becomes negligible. An example of such behavior would be a situation where a $\mathrm{He}$ atom moves quickly between a few closely spaced shallow adsorption potential well minima, leading to an averaged blocking of the tunnel current. Because of an increase of the electron wind force, the average time spent in each of the local well minima may change, until a situation is reached where the $\mathrm{He}$ is pinned to only one minimum. A simple calculation based on this model shows that it indeed leads to a behavior which resembles the experimental result, but to get quantitatively a good correspondence, an unrealistically high scattering cross section with a radius of a few $\AA$ has to be assumed.

Concluding, we have observed a strong distortion of the tunnel resistance as a function of electrode separation tighten when measurements are performed in ${ }^{3} \mathrm{He}$ or ${ }^{4} \mathrm{He}$ environment at low temperatures. The effect can be described using a model that combines a gradual change of the He adsorption potential well for the two electrodes as a function of electrode separation with a local decrease of the conduction electron state density close to the Fermi level caused by the adsorbed He atom. Two-level tunnel resistance fluctuations were observed over a limited range of electrode separations when the measurements were done in $\mathrm{He}$ gas at $1.2 \mathrm{~K}$. These fluctuations may be related to the spontaneous tunneling of a $\mathrm{He}$ atom between the two electrodes.
Part of this work was supported by the Stichting voor Fundamenteel Onderzoek der Materie (FOM) which is financially supported by the Nederlandse Organisatie voor Wetenschappelijk Onderzoek (NWO). We thank J. G. H. Hermsen, J. W. Gerritsen, and E. J. G. Boon for technical and computational support, and I. K. Yanson, S.I. Shevchenko, and D. M. Eigler for stimulating discussions. O.I.S. wishes to acknowledge the NWO for a visitor's grant.

[1] D. M. Eigler, P.S. Weiss, E. K. Schweizer, and N. D. Lang, Phys. Rev. Lett. 66, 1189 (1991).

[2] D. M. Eigler and E. K. Schweizer, Nature (London) 344, 524 (1990).

[3] N. D. Lang, Surf. Sci. 299/300, 284 (1994).

[4] N. D. Lang, Phys. Rev. Lett. 56, 1164 (1986).

[5] See, e.g., J.W.G. Wildöer, A.J.A. van Roy, H. van Kempen, and C. P. J. Harmans, Rev. Sci. Instrum. 65, 2849 (1994); D. N. Davydov, R. Deltour, N. Horii, V. A. Timofeev, and A. S. Grokholski, ibid. 64, 3153 (1993); A. P. Fein, J. R. Kirtley, and R. M. Feenstra, ibid. 58, 1806 (1987).

[6] C. J. Muller, J. M. van Ruitenbeek, and L. J. de Jongh, Physica (Amsterdam) 191C, 485 (1992).

[7] H. van Kempen and O. I. Shklyarevskii, Fiz. Nizk. Temp. 19, 816 (1993) [Sov. J. Low Temp. Phys. 19, 583 (1993)].

[8] R. J.P. Keijsers, J. Voets, O. I. Shklyarevskii, and H. van Kempen (to be published).

[9] J. M. Krans, C. J. Muller, I. K. Yanson, Th. C. M. Govaert, R. Hesper, and J. M. van Ruitenbeek, Phys. Rev. B 48, 14721 (1993).

[10] P. Nordlander and J. Harris, J. Phys. C 17, 1141 (1984).

[11] J.F. Annet and P. M. Echenique, Phys. Rev. B 34, 6853 (1986).

[12] D. M. Eigler, C.P. Lutz, and W.E. Rudge, Nature (London) 352, 600 (1991).

[13] J. J. Sáenz and N. García, Phys. Rev. B 47, 7537 (1993).

[14] M. Sumetskii and A. A. Kornyshev, Phys. Rev. B 48, 17493 (1993). 